\title{
Origin of nonthermal emission from the Fermi bubbles and mechanisms of particle acceleration there
}

\author{
V. A. Dogiel $\dagger$, K.-S. Cheng ${ }^{2} \ddagger$, D. O. Chernyshov ${ }^{1}$ and C.-M. Ko ${ }^{3}$ \\ ${ }^{1}$ P. N. Lebedev Institute of Physics, Leninskii pr. 53, 119991 Moscow, Russia \\ email: dogiel@lpi.ru, chernyshov@lpi.ru \\ ${ }^{2}$ Department of Physics, University of Hong Kong, Pokfulam Road, Hong Kong, China \\ email: hrspksc@hku.hk \\ ${ }^{3}$ Institute of Astronomy, National Central University, Chung-Li 32054, Taiwan \\ email: cmko@gm.astro.ncu.edu.tw
}

Introduction. The discovery of the two giant $\gamma$-ray lobes (Fermi bubbles) in the Galactic center (see Dobler et al. 2010 and Su et al. 2010) was one of the most impressive events of the last few years in astrophysics. However, some indications on giant structures in the Galactic center (GC) were observed several years before by WMAP in the radio frequency range between 23 and $33 \mathrm{MHz}$ (Finkbeiner 2004), and by ROSAT in hard X-rays (Bland-Hawthorn \& Cohen 2003). Recent observations performed by the Planck Collaboration (Ade et al. 2012) found also lobes in the microwave range which spatially coincided the Fermi bubbles that indicated on the common origin of these phenomena.

Parameters of emission from the Fermi bubbles have several remarkable distinction:

(a) The structures are symmetrically elongated in the direction perpendicular to the Galactic plane;

(b) Spectra from the nonthermal emission from the bubbles are harder than anywhere in the Galaxy;

(c) The spatial distribution of emission in the bubbles shows sharp edges of the bubbles;

(d) The surface emissivity is almost uniform inside the bubbles although findings of Hooper \& Slatyer (2013) might indicate on features of the $\gamma$-ray spectrum at latitudes $b \leqslant 20^{\circ}$ which they interpreted as a contribution from the dark matter annihilation nearby the GC.

The origin of the bubbles is actively discussed in the literature. Thus, Crocker \& Aharonian (2011) and Zubovas \& Nayakshin (2012) suggested the hadronic origin of $\gamma$-ray emission from the bubbles, when $\gamma$-ray photons are produced by collisions of relativistic protons with that of the background gas. Alternatively, these $\gamma$-rays can be produced by the inverse Compton scattering of relativistic electrons on background photons (leptonic model) and the same electrons generate radio and microwave emission from the bubbles via synchrotron (see e.g. Su et al. 2010). There may be several sources (processes) which generate electrons in the bubbles:

- In-situ stochastic acceleration by MHD-turbulence nearby the bubble surface (Mertsch \& Sarkar 2011);

- Acceleration by shocks which result from periodical star accretion onto the central black hole (Cheng et al. 2011);

$\dagger$ V. A. Dogiel thanks for grants from the IAU and RFFI (grant 12-02-00005) that gave him an opportunity to participate in the IAU303 Symposium.

$\ddagger$ KSC is supported by a GRF grant under HKU 701013 . 
- Acceleration within jets near the GC about $\sim 10^{6}$ yr ago, and subsequent electron transfer into the bubble by convective flows (Guo et al. 2012 and Yang et al. 2013). Below we discuss some of these models.

Stochastic acceleration from the background plasma. In order to reproduce the spatial distribution of $\gamma$-ray emissivity in the bubble Mertsch \& Sarkar (2011) assumed arbitrarily that: a) the acceleration is nonuniformly distributed inside the bubbles and its efficiency increases near the shock which excited the MHD-turbulence inside the bubble; b) the maximum energy of electrons is a function of the distance to the shock.

There are no other evident sources for electrons accelerated in the halo except electrons from the background plasma and electrons injected by supernova remnants (SNRs) or jets. Estimates of acceleration efficiency in the case of stochastic (Fermi) acceleration from the background plasma is not trivial. As Wolfe \& Melia (2006) and Petrosian \& East (2008) showed, the energy supplied by sources of stochastic acceleration is quickly dumped into the thermal plasma because of ionization/Coulomb energy losses of accelerated particles. As a result this acceleration is accompanied by plasma overheating while a tail of nonthermal particles is not formed, i.e. the effect of stochastic acceleration is negligible.

However, latter Chernyshov et al. (2012) concluded that the efficiency of stochastic acceleration depended strongly on parameters of acceleration and prominent tails of nonthermal particles can be generated by the acceleration although the conclusions of Wolfe \& Melia (2006) and Petrosian \& East (2008) are correct for some conditions.

To define whether the stochastic mechanism is able to produce enough accelerated electrons needed for the observed flux of $\gamma$-rays we take the following parameters of the background plasma in the bubbles: the density $n=10^{-2} \mathrm{~cm}^{-3}$ and the temperature $T=2 \mathrm{keV}$ (see $\mathrm{Su}$ et al. 2010 ).

The kinetic equation for the distribution function electrons, $f(p, t)$, when processes of spatial propagation are neglected, has the form

$$
\frac{\partial f}{\partial t}+\frac{1}{p^{2}} \frac{\partial}{\partial p} p^{2}\left[\left(\frac{d p}{d t}\right)_{C} f-\left\{D_{C}+D_{F}(p)\right\} \frac{\partial f}{\partial p}\right]=0,
$$

$(d p / d t)_{C}$ and $D_{C}(p)$ describe particle energy losses and diffusion in the momentum space due to Coulomb collisions. The stochastic (Fermi) acceleration is described as diffusion in the momentum space with the coefficient $D_{F}(p)$, which we take in the form: $D_{F}(p)=$ $\alpha p^{\varsigma} \theta\left(p-p_{0}\right)$ where $\alpha, \varsigma$ and $p_{0}$ are arbitrary parameters.

Parameters of this model can be restricted from the three conditions:

(a) The energy of electrons emitting $\gamma$-rays by inverse Compton is restricted by the value $\sim 10^{12} \mathrm{eV}(\mathrm{Su}$ et al. 2010 and Cheng et al. 2011$)$;

(b) The total $\gamma$-ray flux at energies $E>1 \mathrm{GeV}$ is $F_{\gamma} \simeq 4 \times 10^{37} \mathrm{erg} \mathrm{s}^{-1}$ that restricts the number of accelerated electrons ( $\mathrm{Su}$ et al. 2010);

(c) Mechanism of particle acceleration should effectively generate nonthermal particles i.e . no plasma overheating (Chernyshov et al. 2012).

From the results of numerical calculations shown in Figure 1 we conclude that the stochastic acceleration from background plasma may provide the density and the spectrum of accelerated electrons needed for the observed $\gamma$-ray and radio emission from the bubbles if the parameters of the model are: $\zeta=2.2, \alpha=3.2 \times 10^{-15} \mathrm{~s}^{-1}, p_{0}=(0.2-0.5) \mathrm{mc}$. The required power supplied by sources of acceleration is about $10^{38} \mathrm{erg} \mathrm{s}^{-1}$ that does not exceed the upper limit of the rate of energy release expected in the GC which is in the range from $10^{39}$ (Crocker \& Aharonian 2011) to $10^{41} \mathrm{erg} \mathrm{s}^{-1}$ (Cheng et al. 2011). 

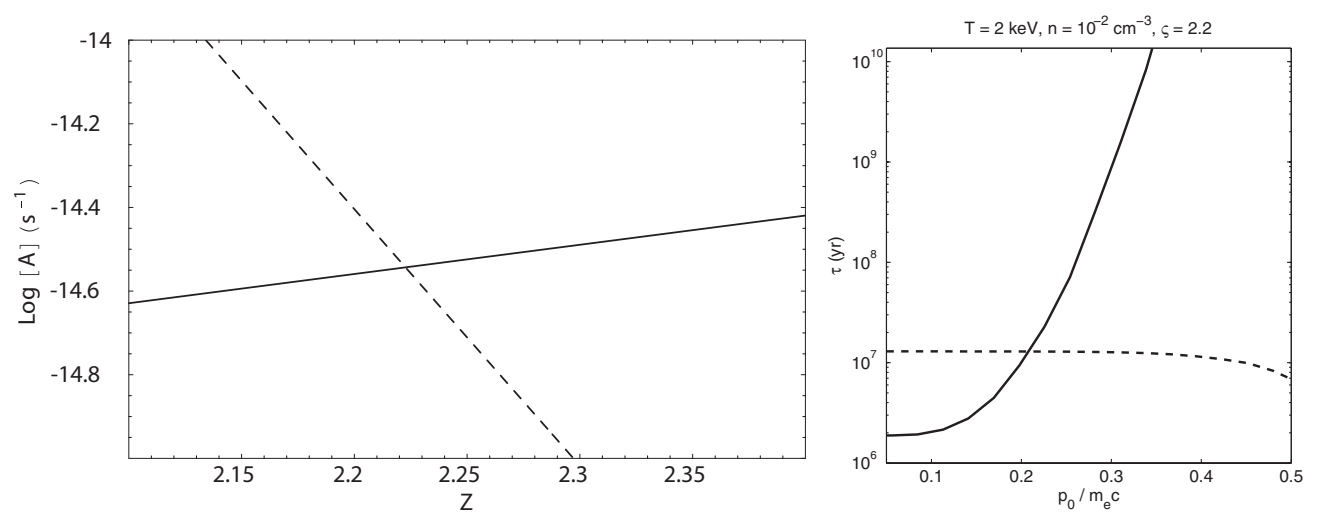

Figure 1. Left panel: The functions of $\alpha(\zeta)$ as derived from the conditions: a) - dashed dotted line; b) - solid line. Right panel: The timescales of temperature variations (solid line) and acceleration (dashed line) for different values of $p_{0}$ (condition $\mathrm{c}$ ).

Stochastic re-acceleration of relativistic electrons emitted by SNRs in the Galactic Disk. In this case the kinetic equation has the form

$$
-\nabla[D(r, z, p) \nabla f-u(r, z) f]+\frac{1}{p^{2}} \frac{\partial}{\partial p} p^{2}\left[\left(\frac{d p}{d t}-\frac{\nabla \mathbf{u}}{3} p\right) f-\kappa(r, z, p) \frac{\partial f}{\partial p}\right]=Q(p, r) \delta(z),
$$

where $r$ is the galactocentric radius, $z$ is the altitude above the Galactic plane, $p$ is the momentum of electrons, $u$ is the velocity of the Galactic wind, $D$ and $\kappa$ are the spatial and momentum (stochastic acceleration) diffusion coefficients, $d p / d t$ describes the rate of electron energy losses, and $Q$ describes the spatial distribution of CR sources in the Galactic plane $(z=0)$ and their injection spectrum.

As it follows from our hydrodynamic numerical simulations the process of re-acceleration of electrons is supposed to take place high above the Galactic plane in regions where the required MHD-turbulence is excited. Therefore because of the synchrotron and inverse Compton energy losses only relatively low energy electrons ejected by SNRs can reach this region. The thickness of re-acceleration region is defined from the intensity of the observed $\gamma$-ray and radio emission.

In the simplest case the number of electrons reaching the re-acceleration region can be calculated in the framework of the diffusion model of CR propagation (see Berezinskii et al. 1990) when the convection terms are neglected $(u=0)$. For calculations we used the model parameters from Ackermann et al. (2012).

Our numerical calculations show that too many high energy electrons are produced in the re-acceleration region and, thus, the condition b) can no be satisfied in the model if the electron spectrum is formed by the acceleration processes only. Formally we can assume that processes of particle escape from the acceleration region are essential enough to make the spectrum steeper and thus to decrease the number of emitting electrons. Indeed, the momentum spectrum of accelerated particles is power-law, $f(p) \propto p^{-\delta}$, with the spectral index $\delta$ equaled

$$
\delta=\frac{3}{2}+\sqrt{\frac{9}{4}+\frac{\tau_{a c c}}{\tau_{e s c}}}
$$

where the acceleration time $\tau_{a c c} \approx \alpha^{-1}$ and escape time is $\tau_{e s c} \approx \Delta r_{b}^{2} / 4 D$. Here $\Delta r_{b}$ is the thickness of re-acceleration region and $D$ is the spatial diffusion coefficient equaled $D(p)=4 v_{a}^{2} p^{2} /(6 \kappa(p))$. Here $v_{a}$ is the Alfven velocity which is about $35 \mathrm{~km} \mathrm{~s}^{-1}$ in the Galactic halo (see Ackermann et al. 2012). The numerical calculations showed that the 
model reproduces the $\gamma$-ray spectrum if $\alpha \sim 10^{-13} \mathrm{~s}^{-1}, \Delta r_{b} \sim 10 \mathrm{pc}$, and $\delta \sim 4$. We notice, however, that the pure diffusion model of CR propagation has serious restrictions. In particular, the effect of convective transfer (Galactic wind) may be essential in the Galaxy as it follows from observations (see e.g. Carretti et al. 2013) as well as from theoretical treatments (Bloemen et al. 1993 and Breitschwerdt et al. 2002). The influence of the wind might decrease the density of SNR electrons in the halo significantly that possibly makes the effect of re-acceleration negligible.

Acceleration by shocks generated by processes of tidal disruption. We discussed this model in details in Cheng et al. (2011). In principle, this model describes quite reasonably the spectra of $\gamma$-ray and radio emission from the Fermi bubbles and it explains the shape of the bubbles because shocks propagate in the exponential atmosphere perpendicular to the Galactic plane (see Kompaneets 1960). However, serious simplifications were used for our calculations, e.g. we used the electron spectrum obtained in a stationary approximation although the situation of shock propagation in the halo is essentially non-stationary, we did not take into account shock evolution in the halo etc. However, we suppose that the shock model of the bubbles does not have serious objections up to now. The energy release from processes of star capture by the central black hole may release a huge energy up to $10^{54} \mathrm{erg}$. A part of this energy is transformed into a flux of hard X-ray emission. Very recently Swift detected to giant X-ray flares in normal galaxies whose luminosity was about $10^{45}-10^{48} \mathrm{erg} / \mathrm{s}$ (see e.g. Bloom et al. 2011). Such a huge flux of hard X-rays from Sgr A* may provide an observational effect in the Galactic molecular clouds seen at present in the form of "Compton echo" (see Cramphorn \& Sunyaev 2002). Besides, some results of observations have been already interpreted as traces of past activity of Sgr A* with a very high energy release (see Bland-Hawthornet al. 2013 and Nakashima et al. 2013) that is in favor of our model.

\section{References}

Ackermann, M., Ajello, M., Atwood, W. B. et al. 2012, ApJ 750, 3

Ade, P. et al. (Planck collaboration) 2012, ArXiv: 1208.5483

Berezinsky V. S., Bulanov S. V., Dogiel V. A., Ginzburg V. L., Ptuskin V. S. 1990, Astrophysics of Cosmic Rays, (ed. V. L. Ginzburg), North Holland

Bland-Hawthorn, J. \& Cohen, M. 2003, ApJ 582, 246

Bland-Hawthorn, J., Maloney, P. R., Sutherland, R. S. \& Madsen, G. J. 2013, ApJ 778, 58

Bloemen, J. B. G.. M., Dogiel, V. A., Dorman, V. L., \& Ptuskin, V. S. 1993, A 6 A 267, 372

Breitschwerdt, D., Dogiel, V. A., \& Völk, H. J. 2002, A\&A 385, 216

Carretti, E., Crocker, R. M., Staveley-Smith, L. et al. 2013, Nature 493, 66

Cheng, K.-S., Chernyshov, D. O., Dogiel, V. A., Ko, C.-M., \& Ip, W.-H. 2011, ApJ 731, L17

Chernyshov, D. O., Dogiel, V. A. \& Ko, C.-M. 2012, ApJ 759, 113

Cramphorn, C. K. \& Sunyaev, R. A. 2002, A\& A 389, 252

Crocker, R. M. \& Aharonian, F. 2011, Phys. Rev. Lett. 106, id.101102

Dobler, G., Finkbeiner, D. P., Cholis, I. et al. 2010, ApJ 717, 825

Finkbeiner, D. P. 2004, ApJ 614, 186

Guo, F., Metheus, W. G., Dobler, G. \& Oh, S. P. 2012, ApJ 756, 182

Hooper, D. \& Slatyer, T. R. 2013, arXiv: 1302.6589

Kompaneets A. S., 1960, Akademiia Nauk SSSR, Doklady (DoSSR, in Russian), 130, 5

Mertsch, P. \& Sarkar, S. 2011, Phys. Rev. Lett. 107, 1101

Nakashima, S., Nobukawa, M., Uchida, H. et al. 2013, ApJ 773, 20

Petrosian, V. \& East, W. E. 2008, ApJ 682, 175

Su, M., Slatyer, T. R., \& Finkbeiner, D. P. 2010, ApJ 724, 1044

Wolfe, B. \& Melia, F. 2006, ApJ 638, 125

Yang, H.-Y. K.aren, Ruszkowski, M., \& Zweibel, E. 2013, MNRAS 2432

Zubovas, K. \& Nayakshin, S. 2012, arXiv: 1203.3060 